\title{
Evaluation of Shielded Enclosure Leak Detection System (SELDS) with Non-Conventional Excitation for Shield Weld Quality Monitoring
}

\author{
Paul Nielsen \\ U.S. Army Engineer \\ Research and Development Center \\ Construction Engineering Research \\ Laboratory, \\ Champaign, IL, USA \\ paul.h.nielsen@erdc.usace.army.mil
}

\author{
William Croisant \\ U.S. Army Engineer \\ Research and Development Center \\ Construction Engineering Research \\ Laboratory, \\ Champaign, IL, USA \\ w.croisant@ieee.org
}

\author{
Michael Mclnerney \\ U.S. Army Engineer \\ Research and Development Center \\ Construction Engineering Research \\ Laboratory, \\ Champaign, IL, USA \\ mkmcinerney@ieee.org
}

\begin{abstract}
In this paper, we describe laboratory evaluations of nonconventional Shielded Enclosure Leak Detection System (SELDS) test techniques for determining/monitoring welded seam quality for EMI/RFI shielded construction. These techniques involve a "transmission line" seam excitation rather than the usual "circuit" excitation. The method of evaluation and results are presented.
\end{abstract}

Keywords

Shielded enclosure leak detection system, SELDS, shielded enclosure test methods

\section{INTRODUCTION}

Large, high performance electromagnetic shielded structures are typically constructed of all welded sheet steel (1/8 to $1 / 4$ inch thickness). Conventional radiated shielding tests cannot be conducted on a shielded enclosure until the enclosure is complete and can be closed on all sides. Completion of the enclosure is usually associated with placement of interior floor treatments. These generally prevent ready access to floor shield welds, which may be shown to be defective by the shielding tests. Seam weld quality is directly related to shielding performance of the completed structure. Thus monitoring of weld quality during construction and during the facility life is a legitimate augmentation of conventional shielding tests. This problem has been addressed theoretically in the past [1], however the authors are not aware of any practical applications of this particular concept. MIL-HDBK-423 [2] describes three proven Shielded Enclosure Leak Detection System (SELDS) techniques, two that employ a radiated field and one that uses current injection. In this paper we present test results of a proposed fourth technique, one that is a combination of the radiated and injected methods. Excitation wires are placed in close proximity to the outside of the welded seams. For seam evaluation, the wires are individually excited, with the shield being used as the current return path. The internal seam is then scanned with the standard SELDS receiver.
The conventional SELDS tests [3] use an excitation of diagonally opposite comers of the area to be tested, or of the whole structure if it is relatively small. A low frequency signal (typically near $100 \mathrm{kHz}$ ) is injected directly onto the exterior of the shield. For the conventional test, the routing of the excitation cables is also important. Placement of an excitation wire too close to the shield can result in a large imbalance of the radio frequency (RF) current density in the shield, causing shielding measurements to vary greatly. (In the SELDS method, the excitation current is not measured, just the leakage signal. This results in a qualitative evaluation of the shield integrity.) Measurements cannot be made near a current injection point for similar reasons.

As with the conventional SELDS test, the observed signal level for the proposed technique is related to weld quality. However, a general unfamiliarity with the new procedure suggested that laboratory evaluations were necessary to determine the usefulness of the concepts and to determine the possibility of improvement. In addition, a study of a technique using the SELDS for determining electrical conduit integrity using an internal excitation wire was conducted.

\section{Sample Preparation and Test Procedure}

ERDC-CERL owns a high performance shielded structure designed for use as a laboratory test facility. It consists of two separate shielded rooms, each with a "window" to accommodate test items. The opening in the room used for this study measures $2^{\prime} \times 4^{\prime}$. Electrical contact to the structure is by a double row of radio frequency interference (RFI) gasket. The removable test sample is bolted onto the window.

Four test samples were produced by a local metal working shop. The test samples were all ASTM A36 sheet steel, 4'6" x 2'6". The mill scale was ground off of a 3" border to allow good electrical contact when mounted on the shielded room window. The samples were match drilled to a template for mounting by bolts. The samples were: (1) $1 / 8$ " (about 11 gage) - no defect, (2) 1/8" with a $1 \mathrm{~mm} \times 20$ 
cm slot cut horizontally in the center, (3) $1 / 4$ " (4 gage) - no defect, (4) $1 / 4$ " with a $1 \mathrm{~mm}$ x $20 \mathrm{~cm}$ slot cut horizontally in the center. Slots were cut using a water jet technique and were intended to simulate a large weld defect for the purposes of this test. Grid points to be used for taking data were painted on the samples.

The four test samples were mounted individually on the 2 ' $x$ 4' window. The mounting was accomplished by uniformly tightening the bolts to a torque of 175 inch-pounds using a torque wrench.

Radiated shielding effectiveness measurements based on IEEE 299 [4] techniques were conducted at $100 \mathrm{kHz}$ on each of the mounted samples using the following equipment:

$\begin{array}{lr}\text { Signal Generator: } & \text { Tektronix FG501, Function Generator } \\ \text { Amplifier: } & \text { Amplifier Research Model 50A220 } \\ \text { Transmitting antenna: } \quad \text { Electro-Metrics Model SHL-30 } \\ \text { Receiver: } \quad \text { Electro-Metrics Model EMC-25, Mark } 3 \\ \begin{array}{lr}\text { Receiving antenna: } & \text { Empire, Model LP-105 }\end{array}\end{array}$

The measurements were made by placing the transmitting antenna at a point $30 \mathrm{~cm}$ away from the center of the test sample external to the shielded room. A reference reading was taken with the receiving antenna placed $60 \mathrm{~cm}$ in the direction opposite of the test sample. The shielding measurement was made by placing the receiving antenna $30 \mathrm{~cm}$ from the test sample inside the shielded room. The room door was sealed and the received signal level was again noted. The reference reading outside the room was then taken again. Shielding effectiveness is the difference, expressed in $\mathrm{db}$, between the lowest of the reference readings and the reading inside the sealed room.

The results are as follows:

$\begin{array}{ccc}\text { Sample } & \text { Description } & \begin{array}{c}\text { Shielding Effectiveness } \\ (\mathrm{db})\end{array} \\ 1 & 1 / 8 \text { " } & 86+ \\ 2 & 1 / 8 \text { " w slot } & 41 \\ 3 & 1 / 4 \text { " } & 86+ \\ 4 & 1 / 4 \text { " w slot } & 43\end{array}$

The samples without slots had shielding effectiveness values beyond the dynamic measurement range of the instrumentation.

SELDS measurements were then conducted on each sample. The SELDS systems consist of a signal generator, which in conventional use is connected to diagonally opposite corners of a shielded structure in order to drive a 100 $\mathrm{kHz}$ signal on the outside surface. A hand held detector equipped with a sensor probe at the end of a rod is used to "sniff" seams and shield penetrations internal to the room.
Two varieties of SELDS equipment were used in this study: (1) A Singer Model 500 consisting of Generator Model 94443-1 (100 kHz) and Detector 94435-1. The transmitter has no variable controls. The detector had been modified by adding an additional $30 \mathrm{db}$ attenuator, giving the instrument a dynamic range of $110 \mathrm{db}$ compared to the original $80 \mathrm{db}$. The readings are directly proportional (in db) to detected signal level. (2) Camel Labs Test Transmitter (95 kHz) Model CL-105 and Test Receiver Model CL-106. Gain and tuning controls on the transmitter are used to maintain the applied signal at a constant value as shown by the front panel meter reading. A series capacitor may be necessary for the unit to tune satisfactorily. The readings on the receiver are expressed in Shielding Units. This means that the magnitude of the readings is inversely proportional to the signal strength of the leakage signal. A high reading is associated with a low signal level and a low reading is associated with a high signal level.

A third variety of SELDS was ordered, but arrived too late to be used in this study. It is an Antenna Research Associates Model LDS-5140 (also labeled ESTAR 5140). This appears to be an updated version of the no longer available Singer Model 500.

Figure 1 is a sketch of the excitation part of the SELDS test conducted in this study. A \#10 copper wire was placed in a $1 / 2$ " plastic conduit fixed at a distance of 1 " from the surface of the test sample. Horizontal excitation has the wire in parallel with the long dimension of the slot, while in vertical excitation it is transverse to the long dimension. The SELDS generator output was attached to this wire and to the external surface of the shielded room. The terminating end of the copper wire was also attached to the external surface of the room. Horizontal (parallel) and vertical (transverse) excitation measurements were done separately.

The receiver was mounted on an adjustable, movable stand (Figure 2) allowing relatively accurate and repeatable positioning of the receiver sensor coil at fixed locations near the test samples inside the shielded room. Readings were taken to produce linear plots of field strength for field maps. The linear plots result from taking data at $2 \mathrm{~cm}$ intervals along a horizontal path at the elevation of the slot, $2.5 \mathrm{~cm}$ from the panel. Data readings for field mapping were taken in the areas approximately as outlined in Figure 2. Two areas were scanned. One was a vertical plane approximately $1.25 \mathrm{~cm}$ from and parallel to the panel surface. Readings were taken on horizontal lines every $4 \mathrm{~cm}$ vertically, starting at the horizontal line containing the slot and extending upward. Fields were assumed to be symmetrical and most of the data for field mapping were taken only above the slot. The second area scanned was in a horizontal plane perpendicular to the test panel starting with the line $1.25 \mathrm{~cm}$ from the surface and extending into the room. 
This plane contains the horizontal slot. Data were taken in horizontal lines every $2.5 \mathrm{~cm}$ in the horizontal plane extending approximately $15 \mathrm{~cm}$ into the room. Both of these data sets were collected using the $1 / 8$ " plate with a slot. Data were taken using both vertical (transverse) and horizontal (parallel) excitation.

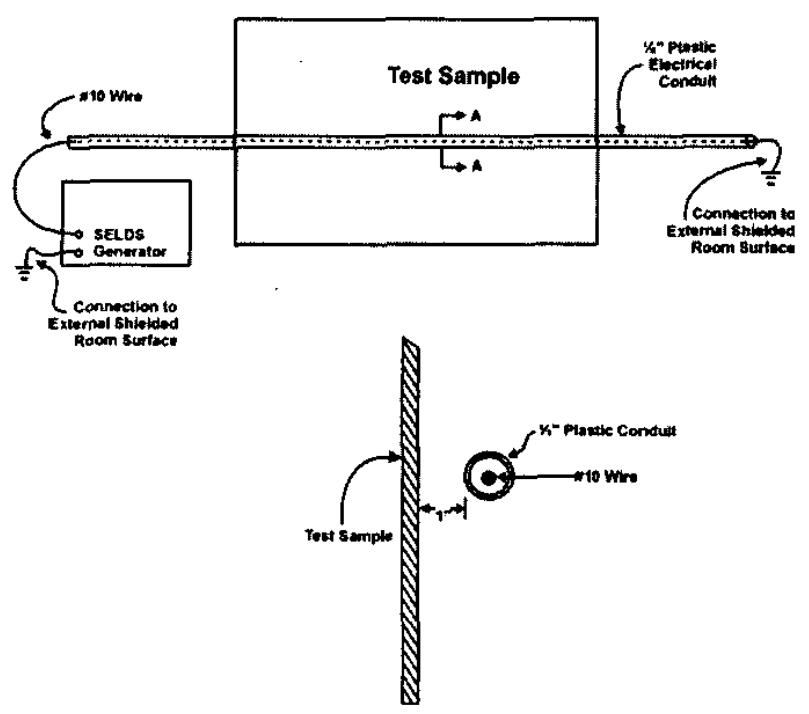

Figure 1. SELDS test configuration for field mapping measurements.

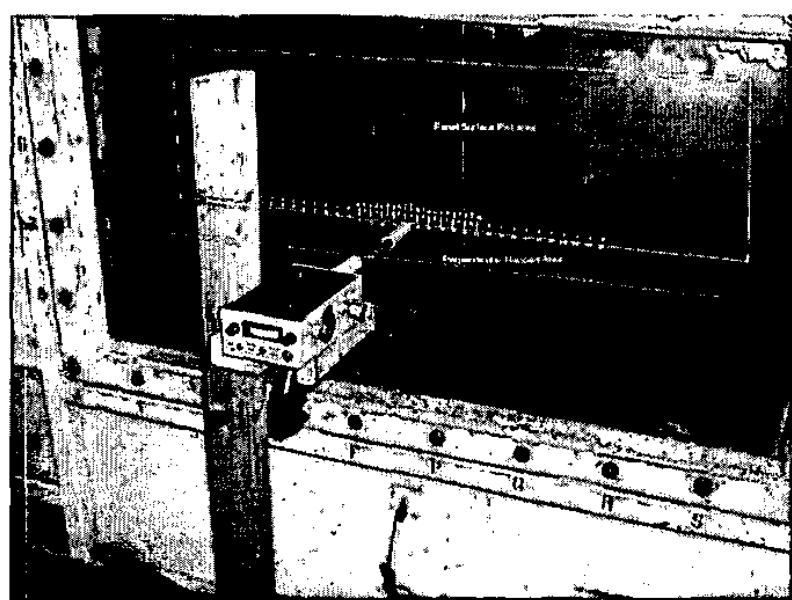

Figure 2. SELDS field mapping with the Carnel Labs Model CL-106 Receiver.

A conventional SELDS measurement was also made using the $1 / 4$ " slotted panel and the Singer system. The driving wires were attached to diagonally opposite corners of the shielded structure containing the test panel. The corners for the attachment were chosen so as to result in nearly diagonal current flow across the slot (Figure 3). These attachment locations should give the highest current den- sity at the slot and the highest leakage signal level from any of the three possible diagonals. Other considerations, such as slot orientation with respect to current stream lines, may be factors, too.

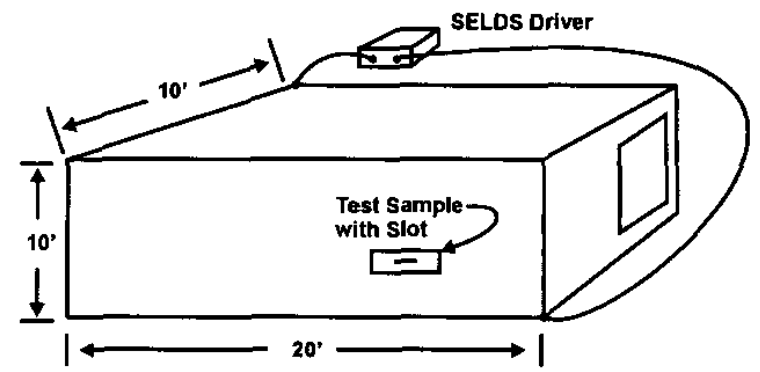

Figure 3. Conventional SELDS test of a shielded structure.

\section{Results and Analysis}

The results of the Singer SELDS measurements, using both the new and conventional excitation methods, along the centerline of the panel for both the 1/8" and 1/4" panels with slots are shown in Figure 4.

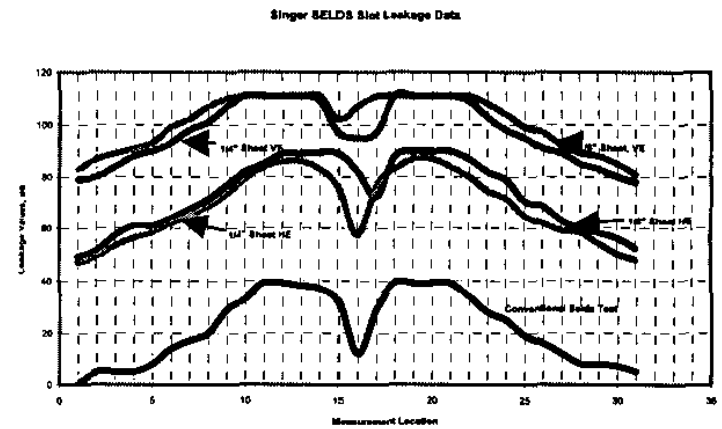

Figure 4. Slot leakage data taken with a Singer Model 500 SELDS. Horizontal, across center of panel. HE=horizontal excitation, VE=vertical excitation. Probe tip $0.125 \mathrm{~cm}$ from surface.

Data taken with the Carnel Labs SELDS for the 1/8" panel appear in Figure 5. This instrument adds another confusion factor in that its readings are in shielding units, essentially the inverse of field strength. Since in this case the interest was in comparison of relative field strengths, the Camel Labs data are plotted by subtracting the measured Shielding Unit numbers from an arbitrary number of 120 . Plotting the Shielding Units without this adjustment would result in an inverted plot relative to the Singer data. 
Observations related to the plotted data include the following:

1. Signal levels were higher for the transverse excitation (approximately $30 \mathrm{db}$ ).

2. For similar excitation, signal levels were higher for the $1 / 8$ " thick sample.

3. The shape of the curve consists of two peak field strength values separated by a minimum reading. The minimum appears approximately in the center of the long dimension of the slot.

4. The information is signal magnitude only. No phase information can be obtained from the SELDS readings.

5. The conventional SELDS measurement resulted in the lowest leakage signal levels measured. This is likely due to the fact that the current density resulting from the diagonal excitation of the total structure is lower than that obtained by a focused excitation with the new technique.

07 Marech Carmet Labs Snittor - 19a" Panel with Blot

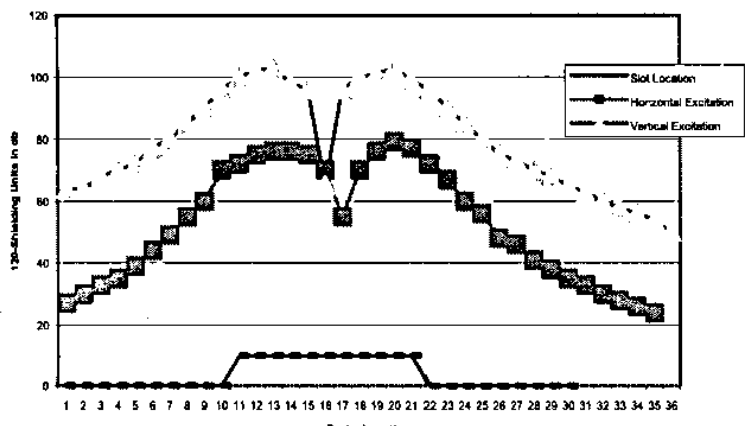

Probe Locestion

Figure 5. Carnel Labs SELDS slot leakage (field strength) data. Horizontal across center of panel.

Enough data to plot 3-D and/or contour field maps were taken. Figure 6 is a plot of data taken with the Carnel Labs SELDS of a 1/4" slotted panel taken in the plane of the slot extending into the shielded room with excitation transverse to the slot. Figure 7 shows data taken in a plane parallel to the test panel.

An additional observation that can be made from the field map plots is that the minimum value, seen near the middle of the slot in the linear plots, extends out to the end of the data taken in both directions - vertically away from the slot and into the room away from the slot.

A measurement suggested by the shape of the field plots consisted of using an oscilloscope to observe the phase of the leakage signal. The availability of two SELDS receiver sensors/probes of each type (Singer and Camel Labs) allowed the oscilloscope to be triggered by a signal from a second probe placed outside the shielded room near the connection to the excitation wire. The oscilloscope thus was triggered at a fixed location on the excitation signal and it was seen that the phase relationship of the signals on opposite sides of the minimum was 180 degrees.

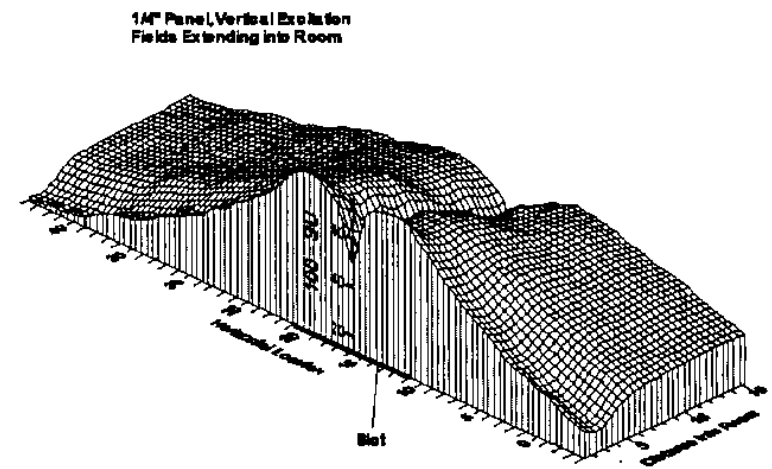

Figure 6. H-Field map of $1 / 4$ " panel data, transverse excitation. Data taken in plane of slot extending into shielded room.

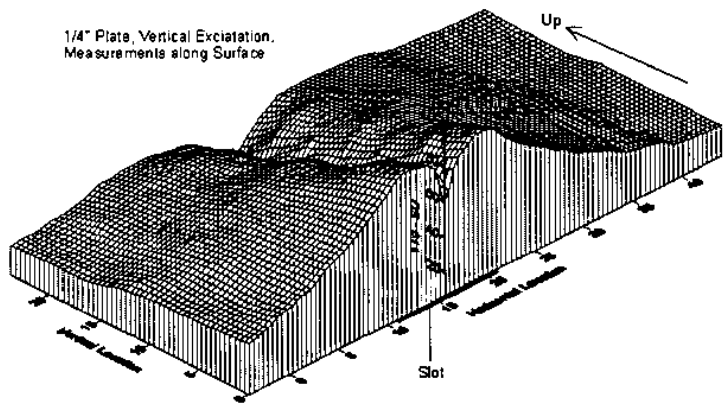

Figure 7. H-Field map of $1 / 4^{\prime \prime}$ panel data, transverse excitation. Data taken parallel to panel surface, above plane of slot.

Similar phase reversals were noted for low level leaks on the periphery of the test sample mounting.

The next step consisted of using a loop antenna to excite the external surface of the shielded room containing the steel plate with the slot with a variety of frequencies (Figure 8). An Electro-Metrics Model SHL-30 12" diameter loop was used for frequencies from about $10 \mathrm{kHz}$ to 5 $\mathrm{MHz}$. At lower frequencies, the impedance of the loop was so low that the signal generator could no longer produce a sine wave. Thus a home made 48 " diameter loop with approximately 25 turns of wire (having survived numerous laboratory clean ups) was used for frequencies from 500 $\mathrm{Hz}$ to $5 \mathrm{kHz}$. Leakage signal observations with the oscilloscope showed similar results as noted above - two peaks in the field strength separated with a minimum and a 180 degree phase difference between the peaks. This was observed over the whole range of frequencies $(500 \mathrm{~Hz}$ to 5 $\mathrm{MHz}$ ) 


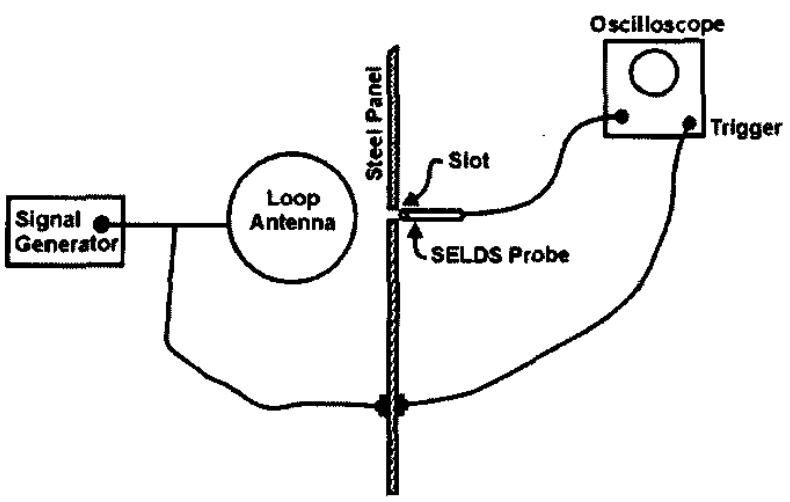

Figure 8. Slot excitation with loop antenna.

Figure 9 shows the phase relationships between signals picked up using two Singer SELDS probes placed at points 13 and 19 of the slot. These are the approximate locations of the peak signal levels.

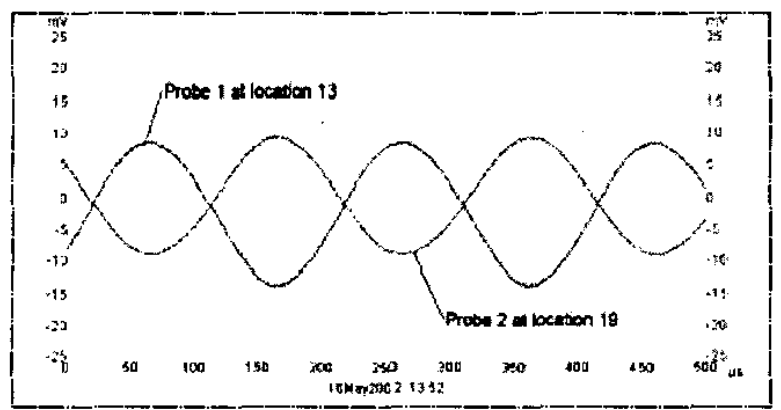

Figure 9. Simultaneous oscilloscope traces of signals measured at Locations 13 and 19 on a slot in a 1/4" thick steel plate using identical Singer SELDS Probes. Frequency $=5000 \mathrm{~Hz}$.

\section{Conclusions}

1. The concept of installing a wire parallel to welded seams is a practical means for getting a sufficient magnitude signal to seam areas for weld quality control during construction. The surface current in the vicinity of a leaky seam will be larger than that obtained by conventional SELDS testing and thus the sensitivity of the measurements is likely to increase. Difficulties in predicting seam locations and complaints from the construction contractor should be expected. A definitive study of the relationship between offset of the drive wire and signal magnitude was not conducted at this time, but would provide useful information.

2. Excitation by a transverse wire over the slot provides a greater magnitude leakage signal than the parallel configuration, but is not practical to implement in a construction project.
3. The field mapping exercise indicates that, large defects, at least, can be detected by "Sniffer" readings at some distance removed. This is important if a concrete slab is installed or for some other reason close access to the shield is not possible after construction.

4. Conventional SELDS measurements appear to result in a significantly lower leakage signal than that which can be obtained with an excitation wire in close proximity to a defect. This is probably due to a lower current density resulting from excitation of the total structure in the conventional approach. Due to transmission line effects, current flow on the shield is concentrated near the wire in the new technique. The new technique should be particularly useful for large structures in which the current density using the conventional procedure will be low and result in reduced sensitivity.

5. This study did not examine the relationship between drive wire placement in relation to a welded seam other than being parallel or transverse. Relatively large displacements are likely to reduce current flow in the seam area and thus decrease sensitivity. In addition, the configuration studies most nearly simulate an unwelded section of butt welded sheets. Other joining configurations (e.g., lap joints) may be of interest.

6. The phase reversal noted at the approximate center of the slot (defect) appears to result from the defect acting as a slot antenna well below resonance.

\section{References:}

[1] Baum, Carl E. (1988), "Monitor for Integrity of Seams in a Shield Enclosure", IEEE Transactions on Electromagnetic Compatibility, Vol. 30, No.3, August 1988.

[2] Military Handbook 423, "High-Altitude Electromagnetic Pulse (HEMP) Protection for Fixed and Transportable Ground-Based C $\mathrm{C}^{4}$ Facilities, Volume I, Fixed Facilities," 15 May 1993, section 17.3.5.1.

[3] Lockwood, R. O. (1967), "New Technique for the Determination of the Integrity of Shielding Enclosures by the Measurement of the Perpendicular Magnetic Field", 1967 IEEE Electromagnetic Compatibility Symposium Record, July 1967.

[4] IEEE Standard 299, "IEEE Standard Method for Measuring the Effectiveness of Electromagnetic Shielding Enclosures," Institute of Electrical and Electronic Engineers. 